\title{
The role of streamwise vorticity in the near-field entrainment of round jets
}

\author{
By DORIAN LIEPMANN† AND MORTEZA GHARIB $\ddagger$ \\ Department of Applied Mechanics and Engineering Science, University of California, \\ San Diego, CA 92093-0411, USA
}

(Received 31 May 1991 and in revised form 26 June 1992)

The role of streamwise vortex structures in the near-field $(x / d<10)$ evolution of a round jet is examined. In free shear layers the streamwise vorticity develops into Bernal-Roshko structures which are streamwise vortex pairs. Similar structures are shown to exist in round jets. These structures, which evolve and amplify in the braid region between primary vortical structures, are shown to drastically alter the entrainment process in the near field and to increase the rate at which fluid is entrained into the jet. As the flow evolves downstream, the efficiency of the streamwise vorticity in entraining fluid increases relative to that of the azimuthal vorticity. Beyond the end of the potential core regime, the entrainment process is mainly controlled by streamwise vorticity. These processes are identified via flow visualization and confirmed by detailed global entrainment measurements.

\section{Introduction}

The fundamental works of Brown \& Roshko (1974) and Winant \& Browand (1974) revealed the central role of two-dimensional vortical structures in the kinematics and dynamics of high-Reynolds-number turbulent mixing layers. Three-dimensional aspects of shear layers were observed shortly afterwards by Konrad (1976), Breidenthal (1981), and Bernal (1981, 1986). Both two- and three-dimensional structures in jets have also been observed by Browand \& Laufer (1975), Yule (1978), Agui \& Hesselink (1988), Liepmann \& Gharib (1989), Liepmann (1990), and Monkewitz et al. (1989). All these works show striking three-dimensional features in coexistence with the primary two-dimensional large vortical structures. The pioneering investigation of turbulent shear flows by Liepmann \& Laufer (1947) had already suggested the existence of a non-planar component of motion.

Konrad's and Briedenthal's flow visualizations of two-dimensional mixing layers clearly showed the presence of a secondary vortex structure in the flow direction. In the planar view, these streamwise structures appeared as thin periodic streaks spaced with a certain spanwise wavelength. Using laser-induced fluorescence (LIF), Bernal (1981) and Bernal \& Roshko (1986) showed that these streaks are vortex pairs that originate in the braid region and fold alternately around the upstream and downstream primary structures. These streamwise structures induced strong undulations in the primary vortex cores and the thin region between them (Breidenthal 1981, Jimenez 1983).

$\dagger$ Present address: Department of Mechanical Engineering, University of California Berkeley, CA 94720, USA.

† Present address: Department of Aeronautics, California Institute of Technology, 1201 E. California Blvd., Pasadena, CA 91125, USA. 
The experimental observations of Lasheras, Cho \& Maxworthy (1986) suggest that the spanwise vortex lines in the braid regions, due to the large strain and vertical shear, respond nonlinearly to small three-dimensional perturbations. Deformation of the spanwise vorticity in the braid region was proposed to be responsible for the further redistribution of the spanwise to streamwise vorticity component which eventually collapses into regions of concentrated vortex pairs (Corcos \& Lin 1984; Lin \& Corcos 1984; Rogers \& Moser 1992). Lasheras et al., while proposing the braids as the location of the first appearance of streamwise vorticity, extended the Bernal-Roshko model to explain the undulations of the primary cores. The computational studies by Ashurst \& Meiburg (1985, 1988) and Martin \& Meiburg (1991) of the streamwise vortices in a mixing layer clearly illustrate the central role of the strain field and vertical shear in the birth and evolution of the streamwise vortices in the braid region.

The issues of the strength and persistence of the streamwise vortices naturally arises when one studies the development of shear layers. The strength and overall impact of these structures on the shear layer is certainly relevant to the entrainment and growth of a shear flow. While the first issue of the strength of the streamwise vortices might be generic to all shear flows, the question of persistence will depend on the particular type of shear layer under investigation.

In the near field, jets can be considered as an axisymmetric shear layer if the velocity profile at the nozzle exit is small relative to the diameter of the orifice. The initial Kelvin-Helmholtz instability develops similarly for both types of flows as does the subsequent rollup of the shear layers into primary vortical structures which appear as a sequence of vortex rings in a round jet. Secondary instabilities also form in both types of flow, which develop into streamwise vortex structures that interact with the primary structures. After this stage in the development of the jet, the potential core region (approximately $x / d<5$ ) ends and the centreline velocity starts to decay. As a result, the velocity difference between the ambient fluid and the highspeed core of the jet decreases and attenuates the shear that supports the vortical rings of the jet. This does not occur in a mixing layer because the velocity difference is held constant. Therefore, the primary structures in mixing layers are continuously supported downstream whereas in jets this support decays. The lack of an imposed constant shear makes the interaction and competition between the azimuthal and streamwise vorticity more dynamic in jets than in mixing layers.

The process of pairing in a round jet as it develops downstream is fundamentally different than its two-dimensional counterpart. In a round jet pairing causes both contraction and expansion of the primary vortical rings and this might enhance the evolution of the secondary, three-dimensional structures. The final destruction of the initially laminar structures at the onset of the mixing transition is strongly influenced by this contraction and stretching process.

It seems that while our understanding of the kinematics and development of streamwise vorticity in mixing layers is fairly comprehensive, some of the more fundamental issues regarding strength, persistence, and overall impact of these structures on the dynamics of the flow is fragmentary; the link between the critical aspects of the flow, i.e. the growth and mixing rates, and the streamwise structures must be established.

Important issues that have not been resolved by previous investigations or exist as unchecked postulates include:

(i) What is the role of streamwise rorticity in the entrainment process of free shear layers? 
(ii) How persistent is the streamwise vorticity relative to the primary twodimensional structures?

This lack of fundamental understanding is due primarily to the difficulty in measuring dynamically significant properties of the flow such as velocity and vorticity. It is difficult, if not impossible, to obtain such properties from smoke or dye visualization. The computational approach, however promising, is still unable to simulate realistic situations where spatially growing shear layers at moderate to high Reynolds numbers are considered.

The recent advances on the quantitative flow visualization techniques such as particle tracking (reviewed in Gharib \& Willert 1989), particle image velocimetry (Adrian 1984), and its digital counterpart (Willert \& Gharib 1991) have made instantaneous global measurements of the velocity and vorticity field possible. Using a novel digital particle image velocimetry technique (DPIV), we were able to investigate the dynamics of streamwise vorticity in shear flows.

In this paper we report on an experimental investigation of the effect of the streamwise vorticity on the near-field entrainment characteristics of a round jet. We have used a combination of flow visualization and velocity measurement (laserDoppler velocimetry and DPIV) techniques to investigate the primary and secondary instabilities and the evolution of streamwise vortical structures in transitional jets. Furthermore we have studied the role of the secondary structures in the near- and far-field entrainment processes of round jets.

Preliminary results from this paper were presented at the IUTAM symposium on mixing in La Jolla, California in August 1990 (Liepmann 1991).

\section{Experimental methods}

\subsection{The jet facility}

The jet nozzle is a smooth contraction following a fifth-order polynomial. The diameter at the exit is $1 \mathrm{in}$. with an area contraction ratio of $36: 1$ and a length-towidth ratio of 1.2. These parameters were chosen to prevent separation during the contraction while making the boundary layer inside the nozzle as thin as possible. The settling chamber and jet are modular (figure 1). As seen in the diagram, water enters the chamber through a perforated tube that injects the fluid in all directions. The large eddies are broken up by two perforated plates. Swirl or axial vorticity is then eliminated by two sets of honeycomb separated by another perforated plate. Three screens perform the final flow conditioning. Each stage is separated by approximately ten characteristic diameters from the next module.

The system is a closed loop: an inline $3.5 \mathrm{in}$. impeller, powered by a 3 h.p. motor, generates the flow. The flow rate is proportional to the speed of the impeller, which is controlled electrically. In each experimental run described below, the exit velocity has been measured directly using laser-Doppler velocimetry (LDV) or been set by adjusting the r.p.m. of the motor. The flow returned through a system of four $2 \mathrm{in}$. pipes spaced evenly along the back of the tank. Each pipe was perforated with small holes spread over $120^{\circ}$; the total area of the holes equalled the cross-sectional area of the pipes. The pipes were connected to a manifold by flexible 2 in. tubes. The manifold led to the impeller. The jet is mounted inside a large glass-walled tank measuring $4 \times 4 \times 8 \mathrm{ft}$. 


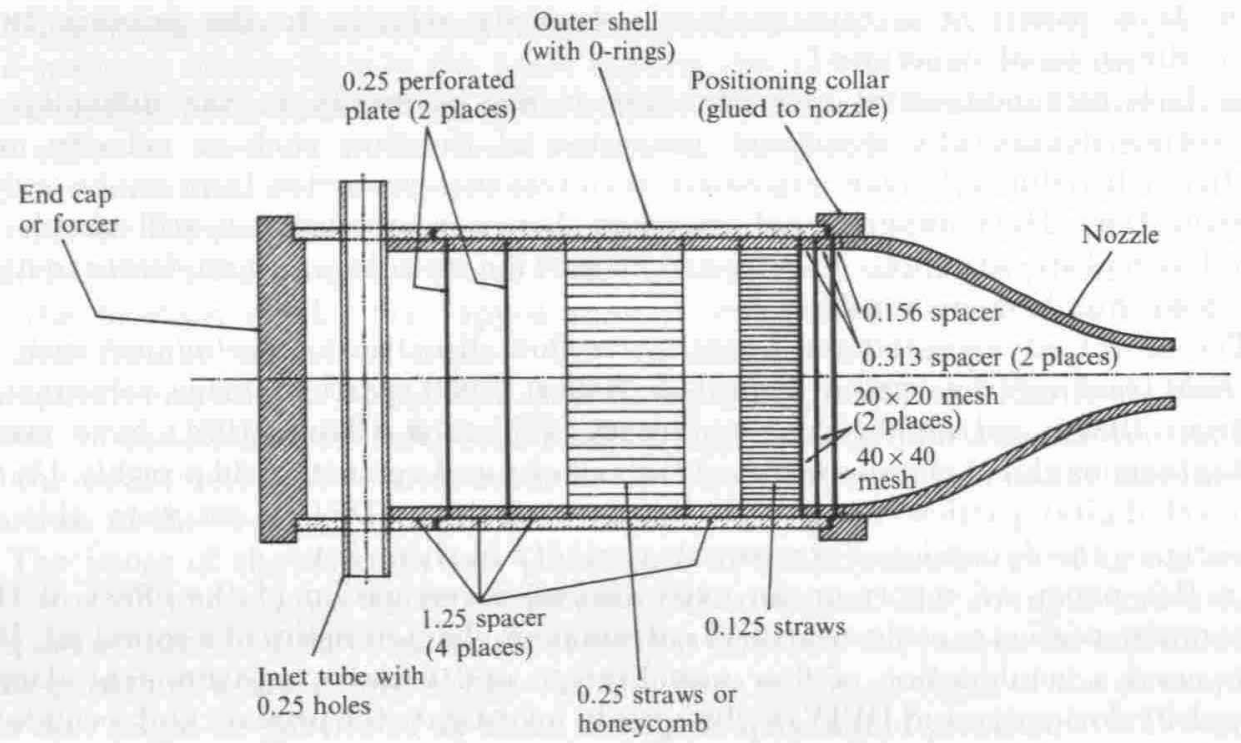

Figure 1. Sehematic of the settling ehamber and jet nozzle (not to seale).

\subsection{Local and global velocity measurements}

Vertical and axial velocity profiles, spectra, and turbulence quantities were measured using a two-eomponent LDV from Dantec operated in backseatter mode. The LDV system has a computer-controlled three-dimensional traversing system which allowed us to map the flow. The tank and jet were seeded with neutrally buoyant, nylon particles with an average diameter of $5 \mu \mathrm{m}$.

Laser-induced fluorescence (LIF) was used to visualize the jet (Dimotakis, MiakeLye \& Papantoniou 1983). Fluorescein dye was injected from a pressure vessel into the jet feed line just before the settling chamber. The dye supply was highly concentrated to minimize the amount of dye dripped into the water so that the jet velocity would not be influenced. The dye was excited using a $5 \mathrm{~W}$ Argon ion laser. For cross-sectional images, the laser light was shot parallel to the tank to a mirror and a scanner mounted on the tank's traverse.

Since the behaviour of a jet is dynamic, only the gross characteristies of the flow field ean be described by statistical point measurements obtained by LDV or hotwire anemometry. The dynamic behaviour requires a measurement technique that is both global and instantaneous. We measured the instantaneous, global entrainment field around a jet using for the first time a new technique developed by Willert \& Gharib (1991). Digital particle image velocimetry (DPIV) provided series of quantitative measurements of the $v$-and $w$-components of velocity as a function of time.

As in most global techniques, DPIV measures the velocity field from the motion of fluorescent particles. Instead of tracking the motion of a few individual particles, as is done in particle tracing techniques, the velocity in each section of the field is determined from the average motion of the particles in a small portion of the field of view, as is done in laser speckle velocimetry or particle image velocimetry; DPIV is the digital counterpart of these methods.

In DPIV, images of the seeded flow are captured digitally at video rates $(30 \mathrm{~Hz})$. Sequential images are then processed to calculate the instantaneous velocity field. 


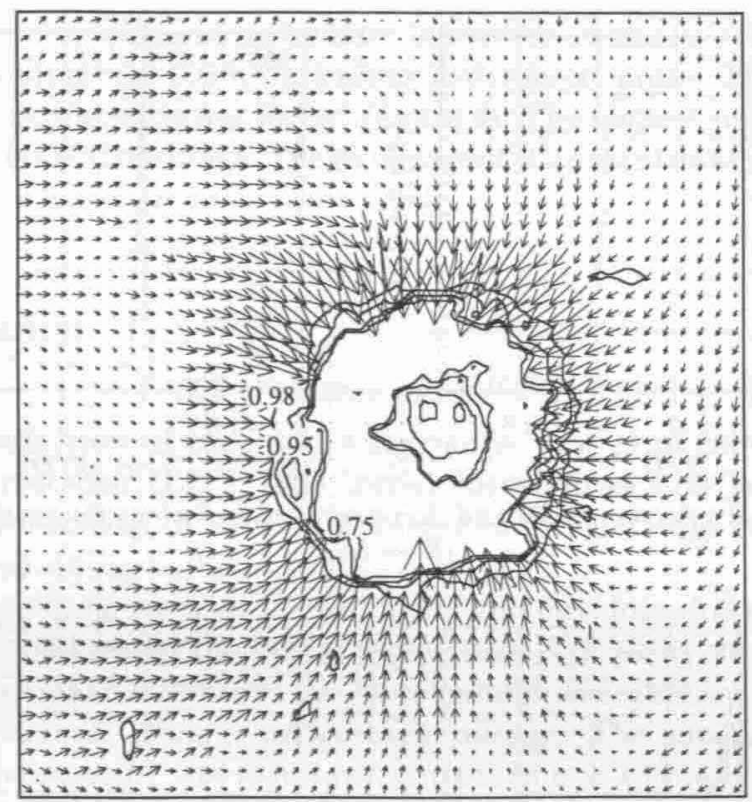

Figure 2. DPIV vector data of the jet in the $(y, z)$-plane (normal to the flow direction). The overlayed contour plot shows the correlation coefficients of the data.

Two $512 \times 480$ pixel fields are subsampled into interrogation windows; the average displacement of the particles imaged in corresponding windows is determined by their spatial cross-correlation. The highest cross-correlation value occurs where the largest number of particle images match with their corresponding spatially shifted partners. The loeation of the correlation peak corresponds to the average displacement of the particles.

In the experiments presented here, the interrogation windows were chosen to be $32 \times 32$ pixels with a $75 \%$ overlap. This resulted in $64 \times 60$ equally spaced velocity vectors per image. The tank was heavily seeded so that there were at least 11 particles per $32 \times 32$ pixel window with each imaged particle about two pixels in diameter. In our experiments, the maximum displacement was approximately 3.4 pixels. The measurement uncertainties corresponding to this displacement at the given seeding density is about 0.05 pixels or $1.5 \%$ (Willert \& Gharib 1990 ). smaller displacements are less uncertain. For a full error analysis see Willert \& Gharib (1991).

The entire tank was seeded with neutrally buoyant polystyrene particles that were coated with fluorescein. The average diameter of the particles was $80 \mu \mathrm{m}$. A series of digital images of the seeded flow field were acquired at $30 \mathrm{~Hz}$ using a three-chip Hitachi video camera linked to a Vicom Image Processing Computer. The optical setup described above for flow visualization was also used for the DPIV measurements except that the laser sheet was thickened to approximately $\frac{1}{4} \mathrm{in}$. A chopper was used to shutter the images in order to avoid particle streaking, which would have reduced measurement accuracy.

In situations where the flow is three-dimensional, data can be lost due to drop-out. However, in these cases the correlation peak is much lower and the spurious data can be filtered out using a threshold criterion. In the DPIV experiments presented in this paper, the flow measurements were made in the $(y, z)$-plane, normal to the axial flow of the jet. In the centre of the jet where the axial velocity is high, the particles move through the laser sheet in less than $\frac{1}{30} \mathrm{~s}$ and the partieles in sequential images are not 

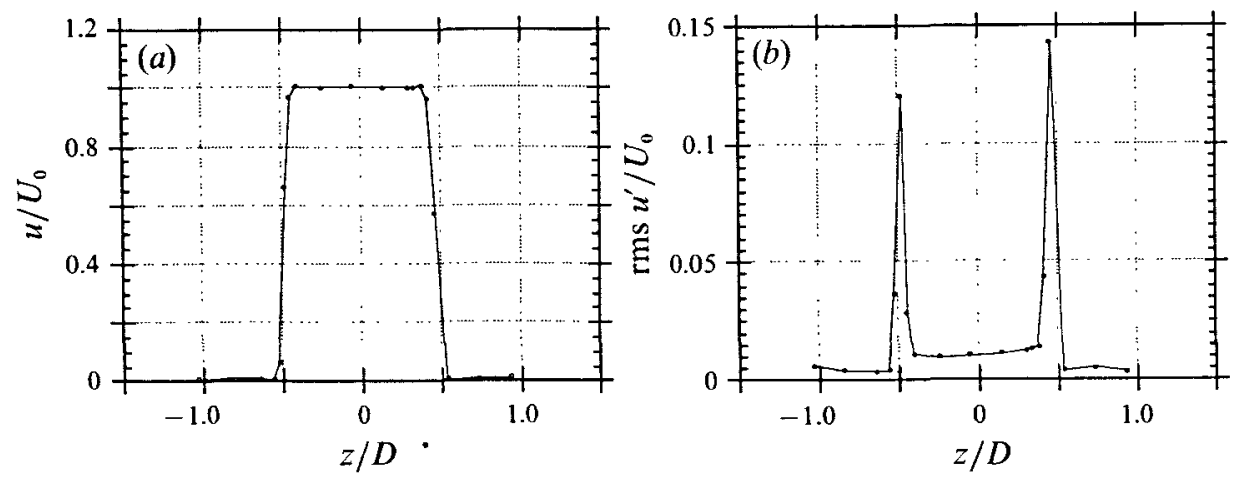

Figure 3. (a) Axial velocity profile and $(b)$ broadband turbulent fluctuations at the jet exit $(R e=5500)$.

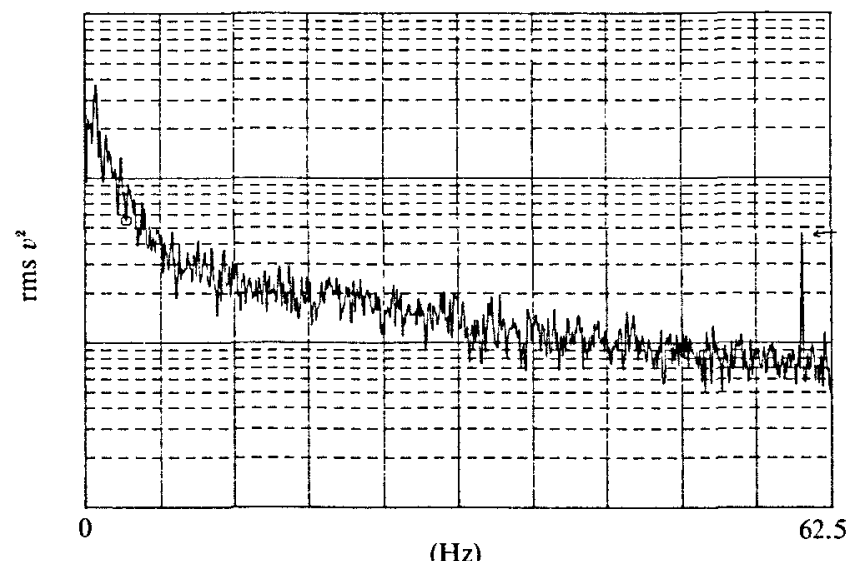

FigURe 4. Velocity power spectrum at the jet exit $(R e=4550$, r.p.m. $=210)$.

correlated. The amplitudes of the resulting correlation peaks are small and the velocity vectors are not plotted because the measurements are not reliable. Figure 2 shows both the correlation coefficient and vector data from the near field of the jet. The vectors with a correlation coefficient of less than 0.95 are not plotted. The correlation coefficients change very rapidly at the edge of the jet indicating that drop-out is not a problem except where there is appreciable flow normal to the measurement plane. Furthermore, the correlation coefficient data indicate that velocity bias errors, resulting from high-speed particles moving through a window too fast to be correlated between sequential images, are not a serious problem in this experiment because this would also significantly reduce the correlation peak size.

The edge of the area in which the velocity vectors have been zeroed corresponds to an axial velocity contour. Around the edge of the blanked area, the same percentage of particles have moved out of the laser sheet before the next image is acquired. The edge, then, is the instantaneous shape of the jet defined by the axial velocity.

\subsection{Flow characteristics of the facility}

Near the exit the jet has a top-hat velocity profile (figure $3 a$ ); the data in this figure were taken as close to the nozzle exit as possible using the LDV. The actual location of the profile was at $x / d=0.03$. The shear-layer thickness is less than $5 \%$ of the 
nozzle diameter. The broadband turbulence intensities, r.m.s. $u^{\prime} / U_{0}$, at the centre of the flow are approximately $0.5 \%$ including instrument noise. The velocity power spectra indicate that the jet is not forced (figure 4). The highest peak, aside from the d.c. component, is $60 \mathrm{~Hz}$ line noise. The experimental apparatus and performance are described in greater detail in Liepmann (1990).

\section{Flow visualization}

\subsection{Primary instabilities}

Figure 5 shows a side view of the jet at a Reynolds number of 5500 visualized using laser-induced fluorescence (LIF). The initial instabilities and subsequent vortex rollup lead to the formation of a series of vortex rings. The rings grow as they move downstream by entraining ambient fluid.

Although not seen in figure 5 , as the jet grows and develops downstream, the rings merge in a pairing process. In the initial region $(x / d<2)$ every two vortices usually pair. If the two upstream structures are close enough together, or one of the vortex rings is relatively small, three vortices will merge. The amalgamation process continues downstream until the resultant vortex ring is so large that its diameter almost spans the radius of the jet. The vortex ring then breaks down very abruptly into smaller structures - usually within half a diameter. The location of the structural breakdown coincides with the end of the visible potential core and the start of mixing transition. After the breakdown, the jet grows linearly with downstream distance.

The dynamics of the vortex rings are best illustrated by following the axial position of several vortex cores over time. The graphs in figure 6 show the evolution of a series of primary vortical structures: downstream distance is plotted as a function of time for fourteen vortical rings. The data were obtained from digitized video tape images. In the plots, the axial locations of the primary vortical structures were followed from the point where they first become visible to the end of the potential core, five diameters downstream. The upper and lower vortex cores (figures $6 a$ and $6 b$ respectively) are symmetric within the first two diameters downstream.

Figure $6(b)$ is the $x-t$ diagram for the lower vortex cores. This graph shows a behaviour which is nearly identical to that of figure $6(a)$. The first three vortex cores merge, then there is a solitary core followed by normal pairing. As long as the flow is axisymmetric, $(a)$ and $(b)$ will remain the same. As the flow develops, however, the rings become unstable relative to each other and begin to tilt so that they are no longer normal to the axial flow direction.

The observed differences between the top and bottom cores indicate that the rings are experiencing extensive distortion. The influence of the growing three-dimensional instabilities could be a factor in this. Specifically, if the streamwise structures are asymmetric, then their effect on the primary structures will also be asymmetric.

\subsection{Three-dimensional instabilities}

In the jets, the primary flow, like plane mixing layers, becomes azimuthally unstable in space and time. Secondary instabilities are visible in the LIF side views of the jet: they appear as fingers of dyed fluid that emerge from the braid region between two vortex cores and stretch around the following vortex (identified by an arrow in figure 5). Previous researches observed these formations as sheaths of turbulent fluid that tended to obscure the visualization of underlying large-scale structures (Browand 




Frgure 5. LIF sideview of the jet at $R e=5500$. The arrow indicates a streamwise strueture at $x / d=3.5$.

\& Laufer 1975 referring to the work of Crow \& Champagne 1971 and Yule 1978). As will be shown below, these secondary structures are streamwise vortex pairs similar to the Bernal-Roshko structures found in mixing layers. Although they can extend outward from the centre of the jet, they are always connected to the main flow. Sometimes, however, they appear as islands of dye because only a thin slice of the flow was illuminated using LIF. These are visible in several flow visualizations experiments (e.g. Dimotakis et al. 1983).

The process by which the three-dimensional instabilities form and develop can be shown by a series of cross-sectional images taken at increasing distances downstream of the nozzle (figures 7-14). These images clearly illustrate the onset of the azimuthal instability and the subsequent development of streamwise vortex pairs. In the figures, the ring structures are shown on the left with the braid regions shown on the right.

The first picture (figure 7) shows the eross-section of the submerged jet at $x / d=1.0$. In this region the primary instabilities have just formed and the jet is completely round. Downstream from this point at $x / d=2.25$ (figure 8) the axisymmetric shear layer rolls up into primary structures. The flow is symmetric; the dark rings are ambient fluid entrained by the axisymmetric rollup of the jet. The solid inner region is the potential core of the jet. The image of the braid region (figure $8 b$ ) shows the presence of small azimuthal instabilities which are absent in the ring cross-section on the left (figure $8 a$ ). This pair of images indicates that the initial instabilities grow in the braid region and are attenuated by the primary rings. The 

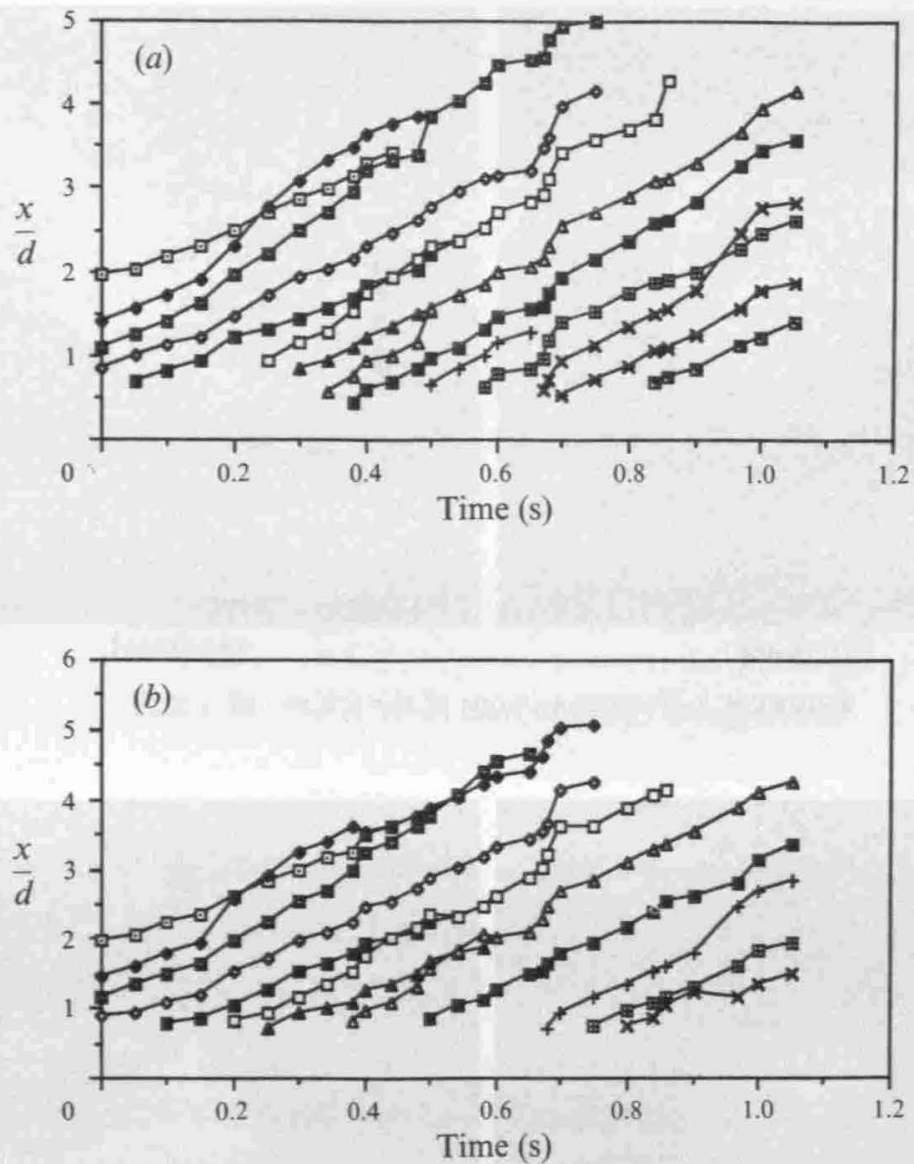

Frgure 6. Axial position of vortex cores versus time for the submerged jet: $(a)$ upper vortex cores, $(b)$ lower vortex cores.

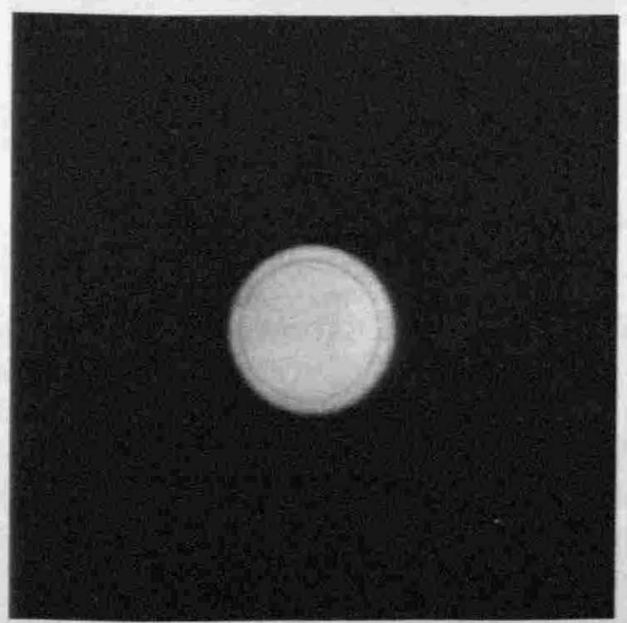

(a) Ring

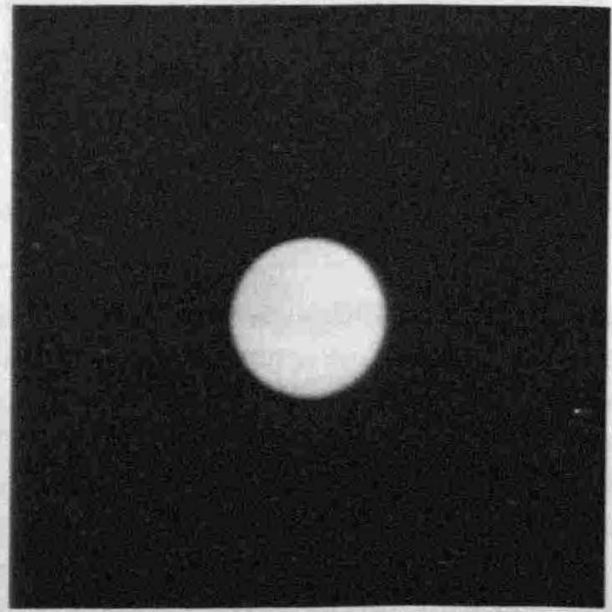

(b) Braid

Froure 7. LIF cross-section of the jet at $x / d=1$. 


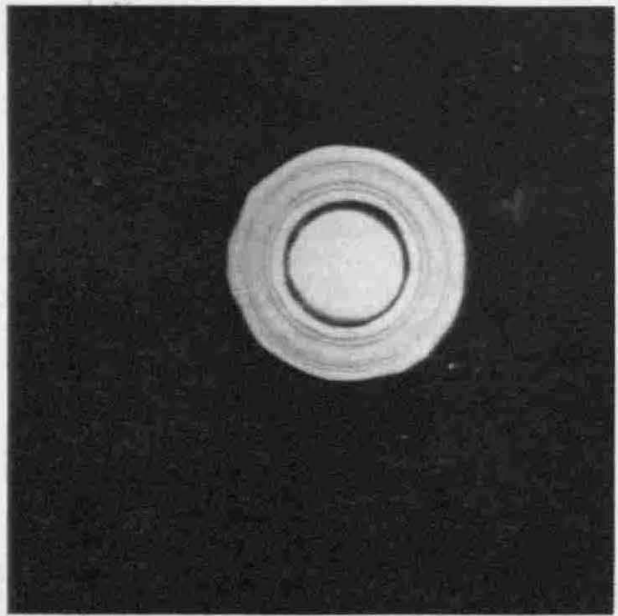

(a) Ring

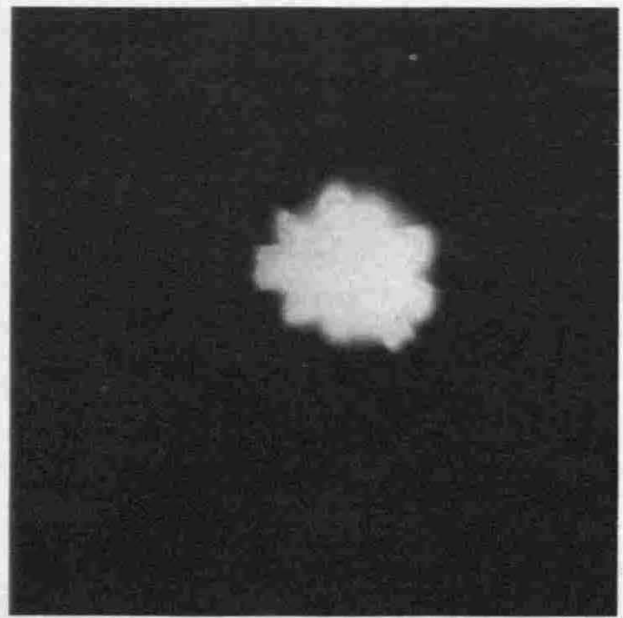

(b) Braid

Figure 8. LIF eross-sections of the jet at $x / d=2.25$.

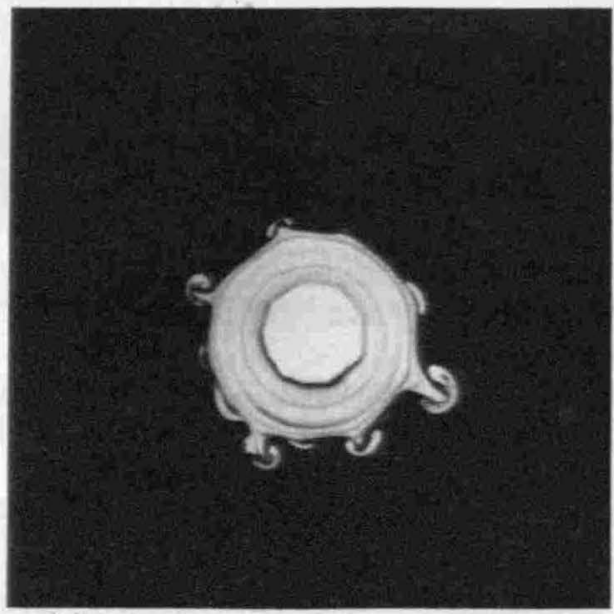

(a) Ring

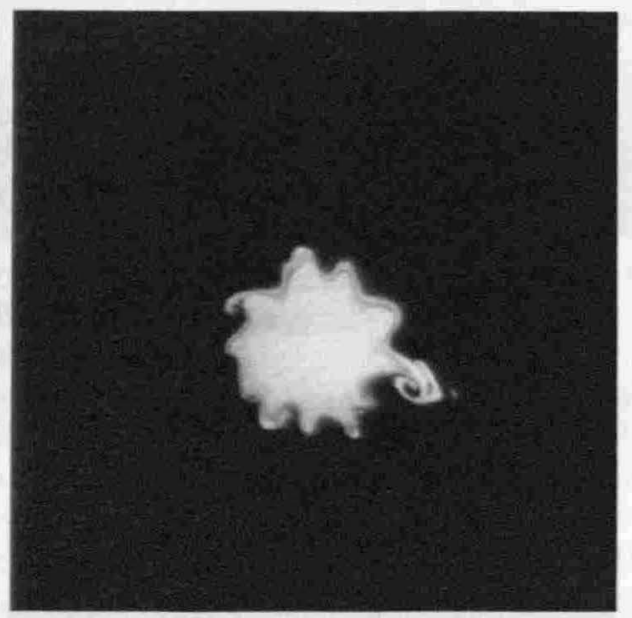

(b) Braid

Figure 9. LIF cross-sections of the jet at $x / d=2.50$.

azimuthal instabilities grow rapidly: figure 9 shows the jet at $x / d=2.50$ where the azimuthal structures are more fully developed. The streamwise structures which are visible around the primary vortical ring in figure $9(a)$, originate from the braid downstream of the image plane as is visible in figure 5 .

Figures 10 and 11 show the continued three-dimensional transition, with streamwise vortex pairs distributed around the jet. These structures move radially in and out with the passage of vortex rings through the laser sheet. The side view in figure 5 also shows the streamwise vortex pairs following the radial motion of the jet. These structures form and then move outward from the jet owing to their induced velocity which is enhanced by rapid stretching. Once they are beyond the high-speed core of the jet, they experience the induced velocity field of the passing primary structures. The radial position of the structures is decided by the balance between 




(a) Ring

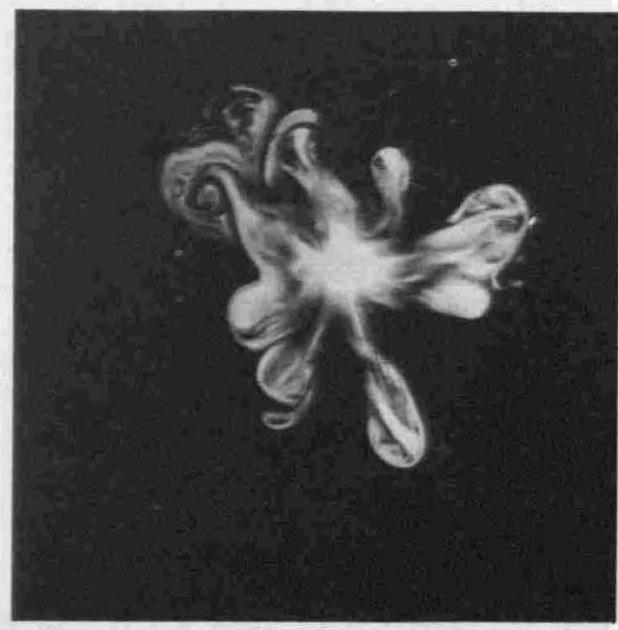

(b) Braid

Figure 10. LIF cross-sections of the jet at $x / d=3.50$.

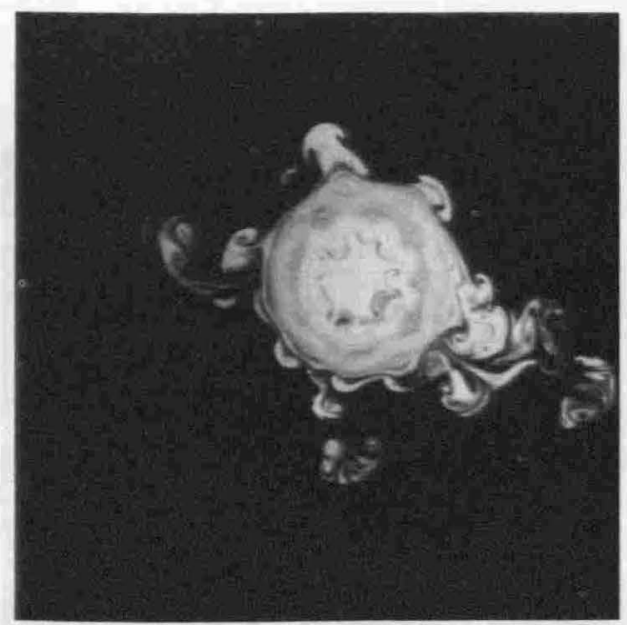

(a) Ring

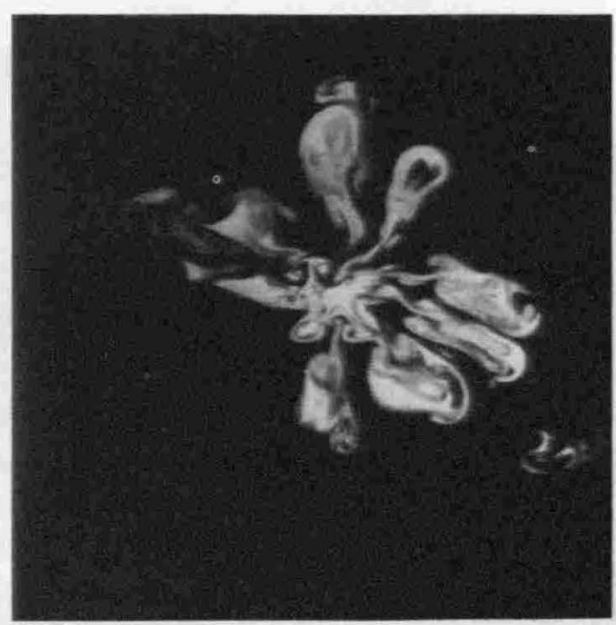

(b) Braid

Figure 11. LIF eross-section of the jet at $x / d=4.00$.

their induced outward velocity and the entraining inward pull of the jet. When they are directly over a braid, they are re-entrained into the jet.

The scenario that the streamwise vortex pairs surrounding the jet in figures 10 and 11 originate from a downstream braid region can be supported by the pair of images in figure 12 , which shows the interaction between the streamwise structures and the primary rings. The sequence oecurs within three video images with the ring following the braid region. The streamwise structures in figure $12(a)$ match the mushroomshaped structures surrounding the ring in figure $12(b)$. The ring exhibits azimuthal instabilities as spatial waves that are both in-plane and out-of-plane with the ring. The waves are out of phase with the streamwise vortex pairs and, therefore, cannot result from the influence of the streamwise vortex pairs surrounding the ring. The streamwise structures in figure $12(b)$, on the other hand, are in phase with the 

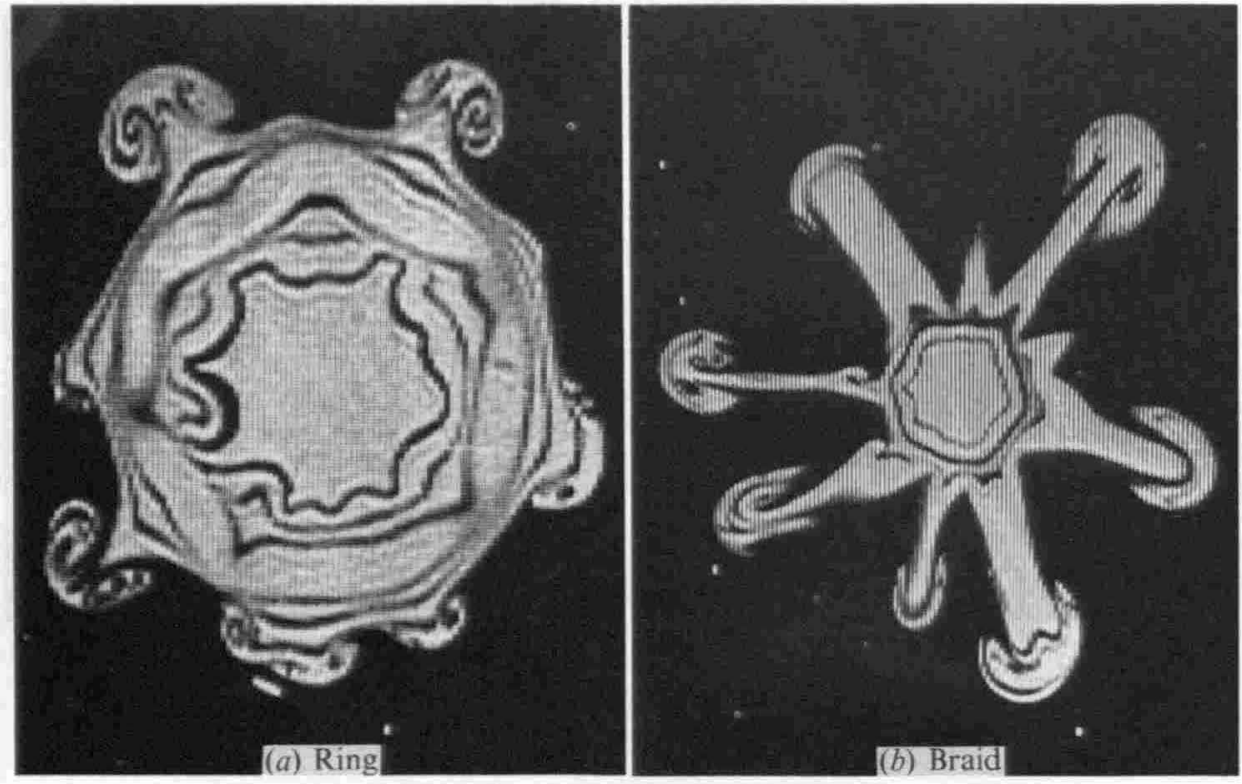

FIGURE 12. LIF cross-section from a video sequence of the jet at $x / d=3.25$ showing the ring and braid region within $\frac{1}{10} \mathrm{~s}$.

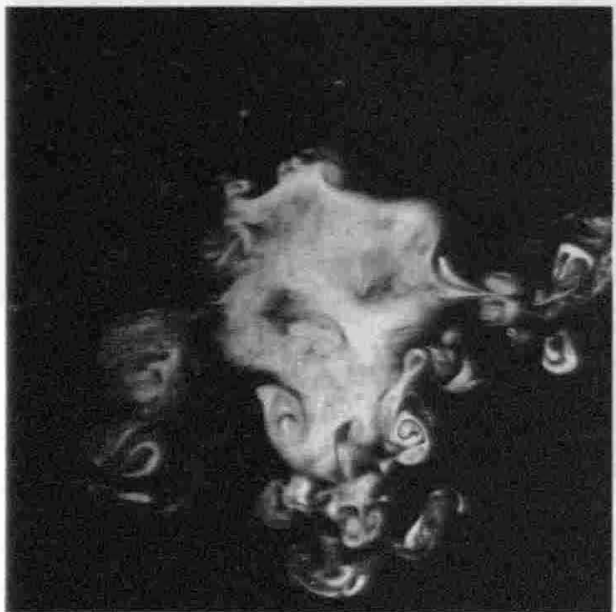

(a) Ring



(b) Braid

FIGURE 13. LIF cross-section of the jet at $x / d=4.75$.

azimuthal instabilities of the potential core which supports the scenario that the streamwise struetures grow out of the braid.

The next set of images (figure 13) are taken near the end of the potential core at $x / d=4.75$. The jet looks much less ordered and more turbulent. At any instant, the jet is never round, but exhibits large structures that are very much a part of the jet structure. The primary structures have become more turbulent and the original ring structure is less apparent (figure $13 a$ ). The streamwise vortical structures have grown and appear to control more of the jet's dynamies (figure 13b). The last image (figure 14) shows the jet at $x / d=10$. The flow is turbulent and there are no 


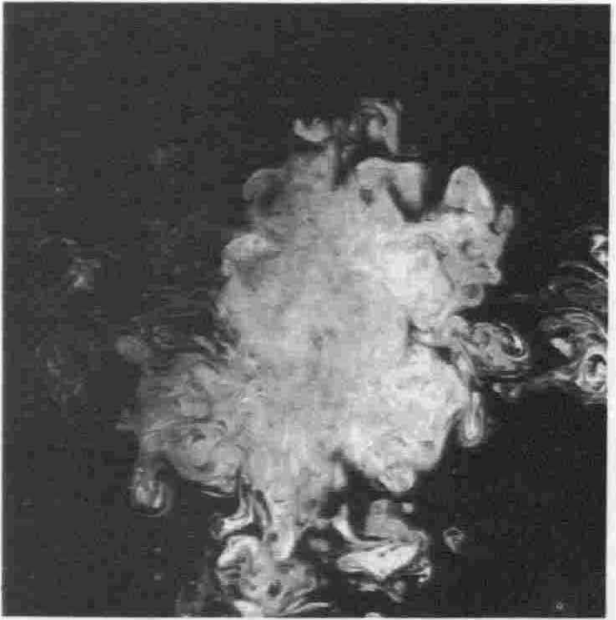

Figure 14. LIF eross-section of the jet at $x / d=10.00$.

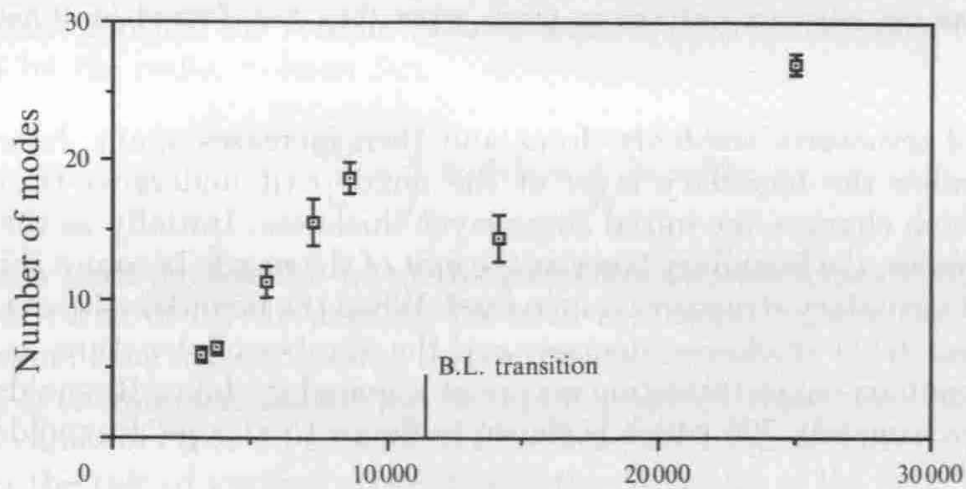

Reynolds number, $U d / \nu$

FIGURE 15. The number of streamwise structures as a function of Reynolds number for a submerged jet. The vertieal line indicates where the boundary-layer Reynolds number $U d / \nu$ is approximately 700 .

recognizable structures left, as a result of the strongly nonlinear interactions in the flow field.

\subsection{Effect of Reynolds number on the streamwise structures}

The effect of Reynolds number on the number of streamwise structures was studied using LIF flow visualization (figure 15). Cross-sectional images of jet with exit velocities from 0.1 to $1.0 \mathrm{~m} / \mathrm{s}$ corresponding to $R e$ of 3000 to 25000 were video taped. For each run, the jet was imaged at sixteen stations from $x / d=0.25$ to 4.0 . The number of streamwise structures was counted at the first station where streamwise structures were first visible rather than at a fixed station for two reasons: first, the downstream location where the secondary structures form decreases with increasing Reynolds number, and second the number of secondary structures decreases with downstream distance. Error bars in the plot show the range of values for all of the runs investigated.

The number of streamwise struetures was found to depend on the Reynolds number of the jet (figure 15). The plot shows that the number of initial streamwise vortex pairs increases with jet velocity. When the Reynolds number reaches 10000 , 


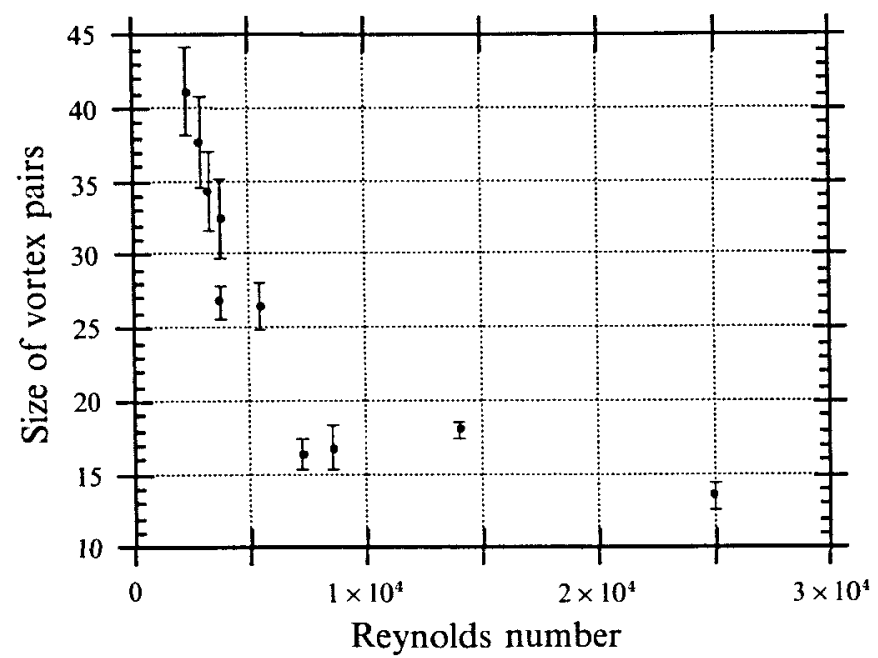

Fig URE 16. The size of streamwise vortex pairs versus Reynolds number. The size of the structures was measured as the intercore distance in pixels when they first formed identifiable vortex pairs.

the number of structures suddenly drops and then increases again. Near this jet Reynolds number the boundary layer at the nozzle exit undergoes transition to turbulence which changes the initial shear-layer thickness. Initially as the velocity of the jet increases, the boundary layer at the exit of the nozzle becomes thinner and the number of secondary structures is increased. When the boundary-layer transition occurs the shear-layer thickness increases and the number of structures is reduced. The nozzle boundary-layer transition occurs at a boundary-layer Reynolds number $(U d / v)$ of approximately 700 which is shown in figure 15 at a jet Reynolds number of 12000 .

Widnall \& Sullivan (1973) found experimentally that vortex rings with a Reynolds number $(\Gamma / \nu)$ of 5790 have only 6 modes. The jet in our experiments at $R e=5500$ had an average of 11 secondary structures which for the primary structures corresponds to a Reynolds number based on their circulation of approximately 4000 . Widnall \& Sullivan also found that the number of modes increases with the Reynolds number, so the number of modes one would expect for a vortex ring at $R e=4000$ would be less than 6 . The difference in the number of modes is an indication that the streamwise structures do not develop from a ring-type instability. This difference supports the results of the flow visualization, where the instabilities first appeared in the braid and not the primary ring (figure 8).

The size of the streamwise structures appears to be inversely proportional to the exit velocity of the jet (figure 16) for $R e<10000$. The size of the vortex pairs was measured as the distance between their cores. The error bars indicate the standard error of the measurements for each Reynolds number. The data indicate that for $R e>10000$ the size of the structures is less dependent on the exit velocity of the jet. It makes sense that the size of the structures would decrease as their number increases as in figure 15, but the real physical significance of the linear scaling for $R e<10000$ and its near constant value for $R e>10000$ is not clear to the authors at this point. 


\section{Global entrainment}

The flow visualization showed the growth of secondary instabilities into streamwise vortex pairs. The side views of the jet indicated that these grew in the braid region of the jet, moved out into the slower moving ambient fluid, and were stretched backward by the axial velocity gradient. The cross-sectional images demonstrated that these structures are, in fact, streamwise vortex pairs similar to the Bernal-Roshko structures in plane shear layers. Even though these structures are clearly evident in the photographs, one can still ask whether they are an active component of the flow field.

First we need to define the concept of entrainment. It is defined by Wygnanski \& Fiedler (1969) and Crow \& Champagne (1971) as the streamwise spatial derivative of the volume flux or $\mathrm{d} Q / \mathrm{d} x$, where

$$
Q=\int_{0}^{2 \pi} \int_{0}^{\infty} u_{x} r \mathrm{~d} r \mathrm{~d} \theta
$$

For constant density, the volume balance requires that the net axial volume flux be balanced by the radial volume flux:

$$
\frac{\mathrm{d} Q}{\mathrm{~d} x}=\oint_{\boldsymbol{c}} \boldsymbol{u} \cdot \hat{\boldsymbol{n}} \mathrm{d} s=\int_{0}^{2 \pi} u_{r} r \mathrm{~d} \theta
$$

where $c$ is a path outside the jet at the given downstream location. If the path is a circle, the value of $\mathrm{d} Q / \mathrm{d} x$ depends on both the radius of the path, the distance downstream of the nozzle, and the instantaneous state of the jet. The far-field value of $\mathrm{d} Q / \mathrm{d} x$ or entrainment should not be time dependent. However, evaluating $\mathrm{d} Q / \mathrm{d} x$ for small values of $r(r \approx d)$, where $u_{r}$ is time dependent, can reveal useful information regarding the role of vortical structures in the dynamics of the local entrainment.

\subsection{The instantaneous entrainment field of the jet}

The velocity vector plots, produced using DPIV, quantitatively describe the velocity field in the $(y, z)$-plane of the jet. As discussed previously, the use of the correlation coefficient data provides a consistent way to delineate regions in the plots where the axial flow is too high for reliable results to be obtained using DPIV. The outer edge of these regions is also an accurate indication of a constant axial velocity contour and, therefore, shows the instantaneous size and shape of the potential core of the jet. Comparisons of the DPIV results with the LDV velocity profiles indicates that the axial velocity is around $5 \mathrm{~cm} / \mathrm{s}$.

The DPIV technique makes it possible to measure the temporal dynamics of the entrainment field around the jet. The flow visualization presented previously showed that near the nozzle, at $x / d=2$, the jet is still round with the dominant structures being vortical rings. The series of DPIV images shown in figure 17 document the passage of a ring through the measurement volume at $x / d=2$. The data shown in figure 17 were taken sequentially: the particle images were acquired at $30 \mathrm{~Hz}$ and two sequential images were processed for each plot so the time between data sets was $66.7 \mathrm{~ms}$. The diagram at the top of each DPIV plot indicates the approximate location of the measurement relative to the primary structures. At the front of the ring the flow induced by the azimuthal vorticity is radially outward (figure $17 a$ ). The shape of the core region, indicated by the lack of velocity vectors, is round and 

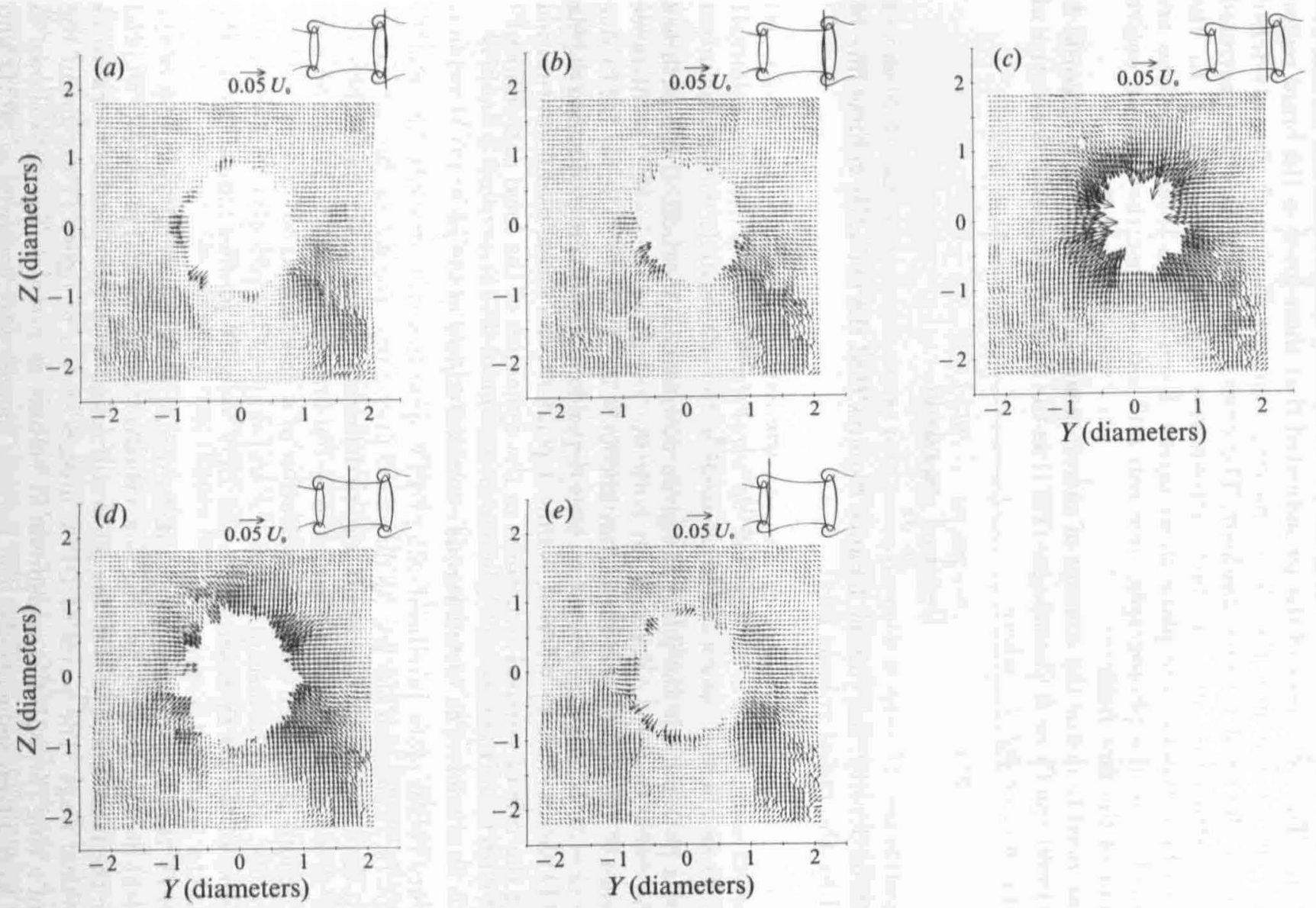

בั

FIGURE 17. Sequence of DPIV data sets showing the evolution of the velocity field in a plane normal to the jet axis at $x / d=2$ during the passage of a primary structure. The small diagrams indicate the approximate location of the sample volume for each 


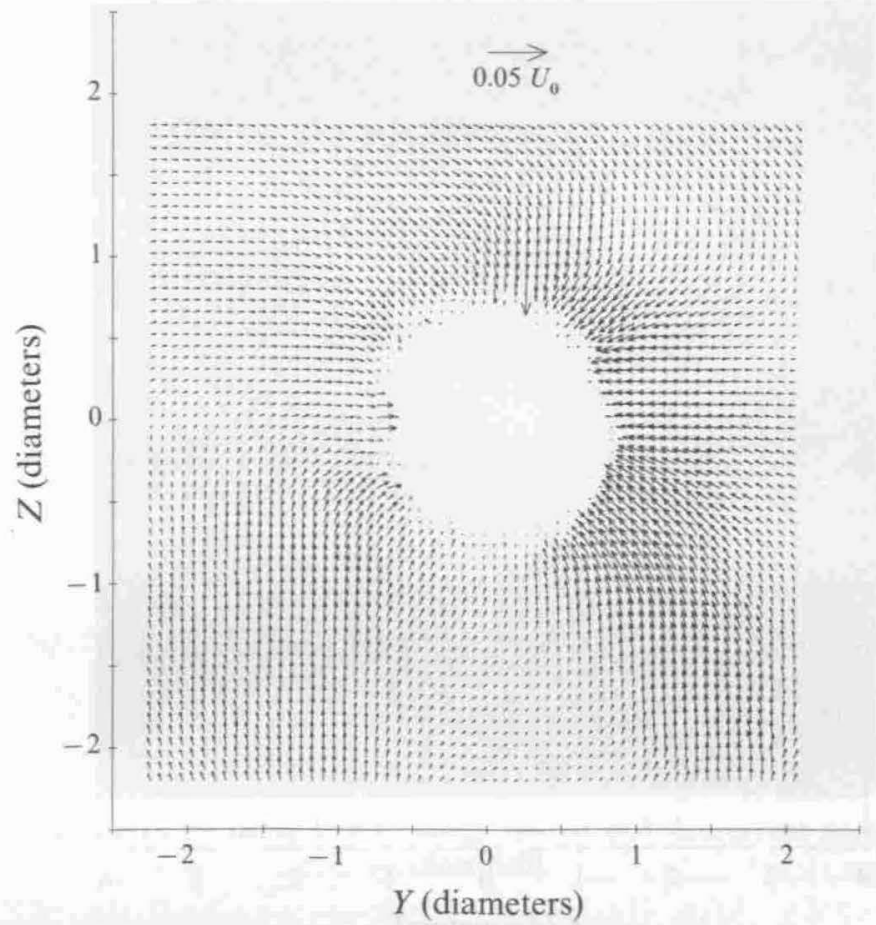

Figure 18. The average velocity field in the plane normal to the jet axis at $x / d=2$.

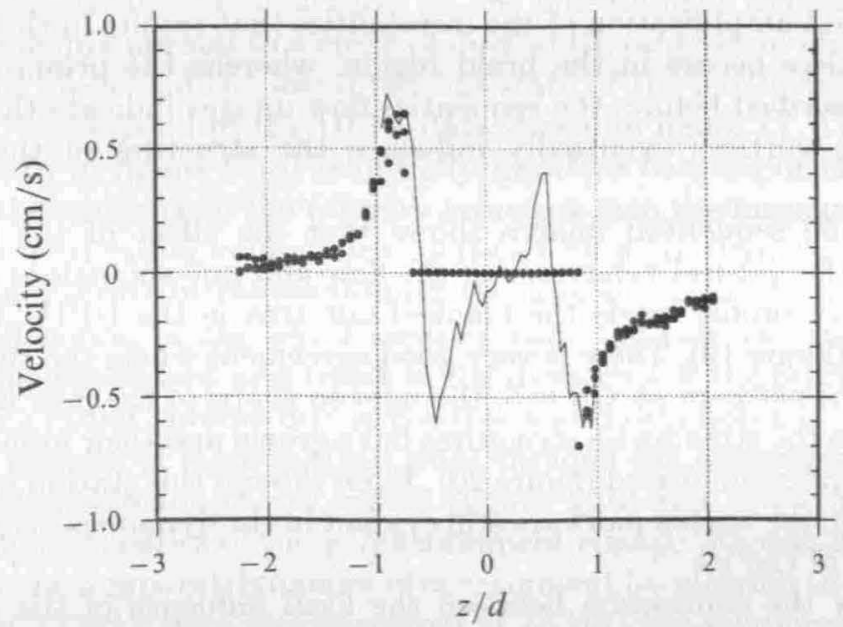

FIGURE 19. Comparison of the vertical velocity profiles at $x / d=2$ obtained via DPIV

and LDV $(\longrightarrow)$.

smooth. As the ring passes, the rate of entrainment increases (figure $17 b$ ). The inward velocity reaches a peak in the braid region between the two vortex rings (figure $17 c, d)$ with the extremum occurring closer to the downstream ring. The final image (figure $17 \mathrm{e}$ ) shows the entrance of the next vortical ring into the measurement volume.

Our flow visualization studies (e.g. figure 8) as well as the DPIV data in figure 17 


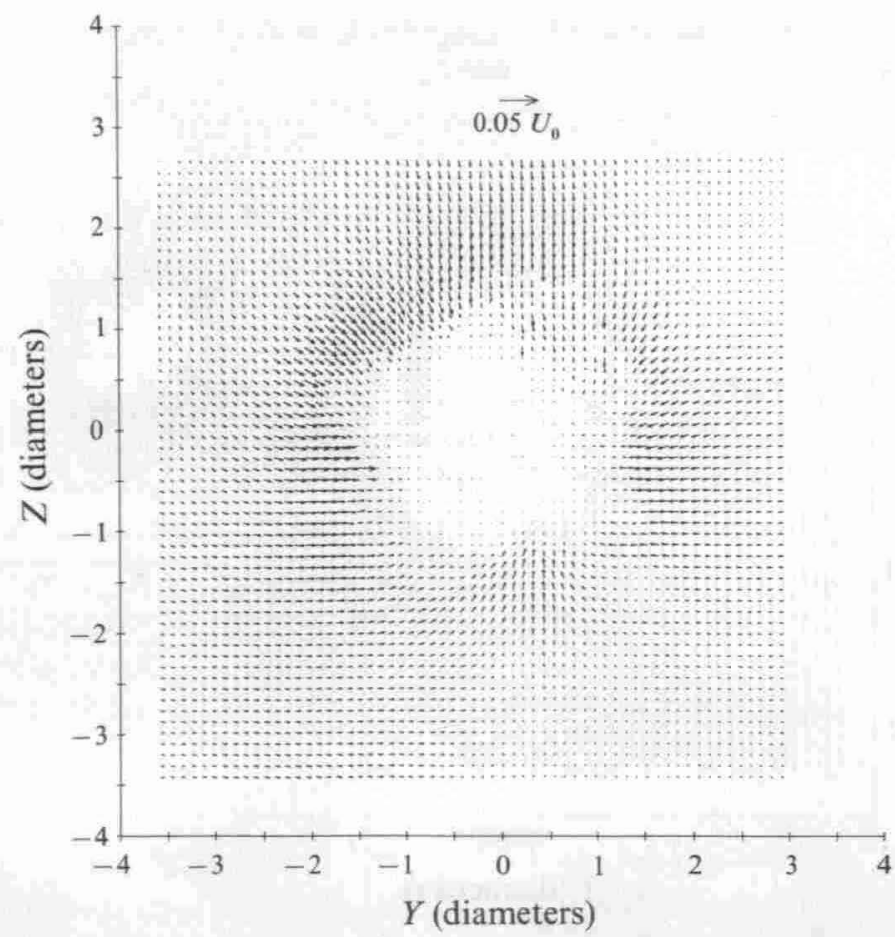

Figure 20. The average velocity field in a plane normal to the jet axis at $x / d=5$.

indicate that the first amplification of the instabilities that results in the generation of streamwise vortices occurs in the braid region, whereas the primary rings are smooth. As was described before, the sequential flow images indicate that the fully formed streamwise vortices eventually influence the structure of the upstream primary ring.

The average of 50 sequential images shows that the effect of the streamwise vortices is part of the natural vehaviour of the flow and not anomalous (figure 18). The average velocity profile inside the blanked out area in the DPIV data can be obtained via LDV (figure 19). There is very good agreement where the two data sets overlap. Farther downstream at $x / d=5$, the uneven shape of the flow field is more pronounced because the streamwise structures have grown and their influence on the entrainment pattern has increased (figure 20). Even though this station is at the end of the potential core, the vortex pairs are still evident in the dynamies of entrainment close to the centre of the jet.

In order to show the connection between the local influence of the streamwise vortex pairs and the velocity field, flow visualization images of the jet were taken with both dye and particles. Figure 21 shows that the longer particle streaks associated with high velocities are concentrated in the areas between the streamwise vortex pairs. It is also apparent that the particles inside the streamwise structures do not have a significant outward velocity. This indicates that for a free jet, the streamwise structures do not behave like the sidejets observed by Monkewitz et al. (1989) in a heated jet. The outward velocity of the natural structures is relatively low. 


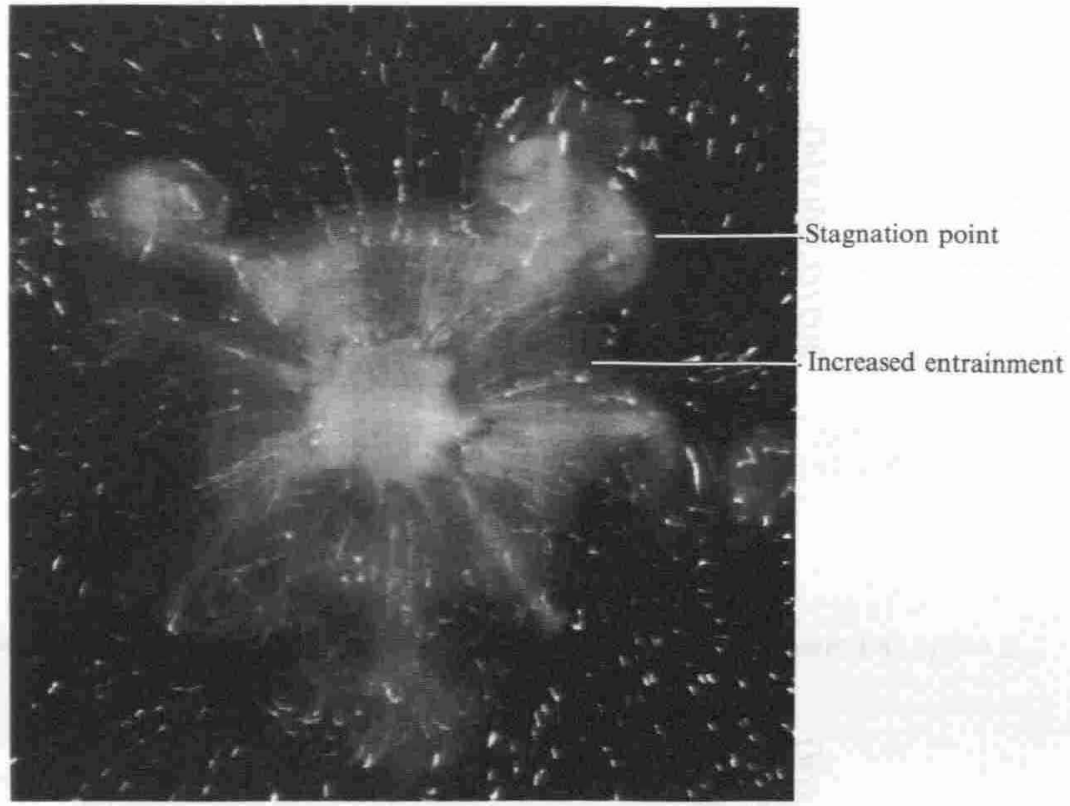

Figure 21. Time exposure using LIF to image the jet and fluorescent particles to image the flow field.

\subsection{The effect of streamwise vorticity on entrainment}

The instantaneous velocity vector information made it possible to determine the impact of the streamwise structures on the entrainment dynamics of the jet. The radial volume flux normal to a eircle of unit width centred on the jet depends on the radius of the circle and the instantaneous state of the jet (figure 22). The curves in figure $22(a)$ correspond to the DPIV data shown in figure 17. The curve calculated from the data in figure $17(a)$ is initially negative because of the outward vectors caused by the entrance of the primary structure into the measurement volume. For $r / d=1$ the local radial volume flux inereases by more than an order of magnitude as the primary structure moves through the laser sheet and the inward flow increases in the braid region of the jet. Therefore, near the core the radial volume flux is strongly time-dependent as a result of the dynamics of the primary structures. For large $r / d$ the radial volume flux is positive and independent of the time variations due to the passage of large vortical rings through the measurement volume.

Farther downstream at $x / d=3.25$ (figure $22 b$ ) and $x / d=5.0$ (figure $22 c$ ) the temporal characteristics of the jet entrainment change. Figures $22(b)$ and $22(c)$, like $22(a)$, show the temporal dynamies of entrainment by plotting the radial volume flux for sequential DPIV data sets measured at $15 \mathrm{~Hz}$. The temporal dynamies near the core of the jet indicate that the relative efficiency of the primary structures at entraining fluid has decreased. Since these structures pass through the measurement volume periodically, their effect at small $r / d$ should manifest itself as temporal fluctuations in the radial flow as in figure $22(a)$. As the jet evolves downstream, the range of these fluctuations decreases (figure $22 c$ ). The tilting of the azimuthal vorticity vectors due to the secondary structures and the resulting breakdown of coherence in the primary structures are responsible for the reduced range of the radial volume flux at small $r / d$. As seen in figure 5 , the streamwise structures stretch backward over the following primary structure and braid region. Local entrainment 

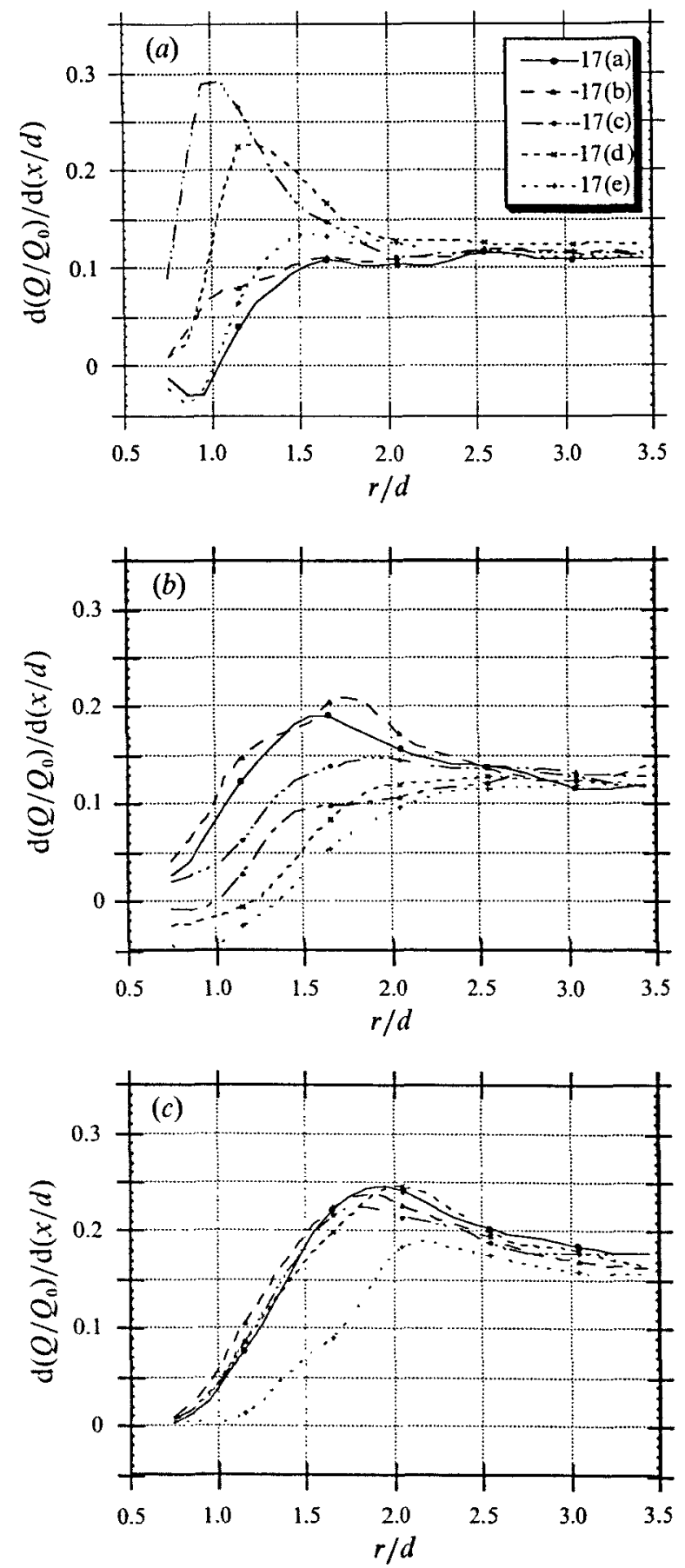

Frgtre 22. The normalized radial volume flux as a function of time and radial distance for the jet at $(a) x / d=2,(b) x / d=3.25$, and $(c) x / d=5$.

due to the secondary structures therefore would not fluctuate with time as much as that due to the primary structures.

These streamwise structures would have a profound influence on the spatial distribution of the radial volume flux for large $r / d$. Such an influence on the 


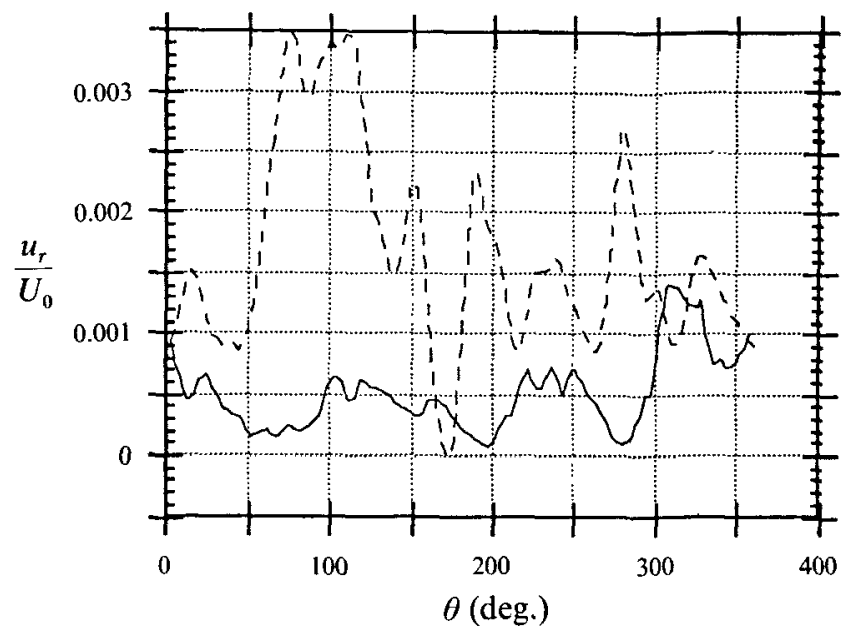

FIGURe 23. Instantaneous inward flow for $x / d=2(-)$ and $5(---)$ measured at $r / d=2$.

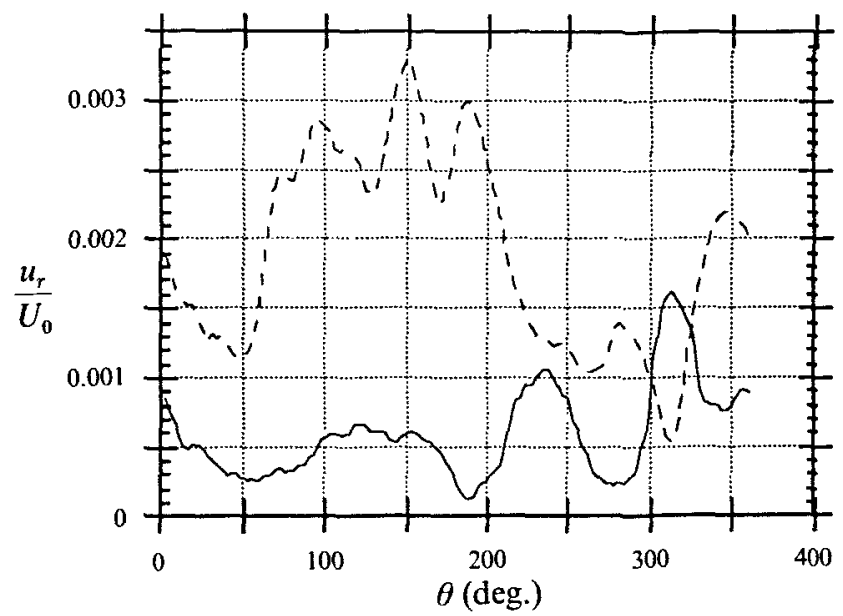

Figure 24. Average inward flow for $x / d=2(-)$ and $5(---)$ measured at $r / d=2$.

entrainment by the streamwise vortices can be seen in figure 23 where the radial velocity is plotted versus azimuthal angle. The plots show that for $x / d=2$ and 5 at $r / d=2$, where the temporal fluctuations due to the passage of large structures have ceased, the azimuthal distribution of the radial velocity has a very distinct wary pattern; an indication of the induced velocity field of the streamwise vorticity. The wavy pattern is not a transient phenomenon since it persists through 50 velocity averages (figure 24 ).

The total radial volume flux at each station can be represented by its value at large $r / d$ where the streamwise velocity component is negligible as defined previously. The radial volume flux is equivalent to the net increase in the total jet volume at each station, i.e. $\mathrm{d} Q / \mathrm{d} x$. It is interesting to note that according to figure $22(a-c)$, while the primary vortices lose their strength, the far-field radial volume flux, or entrainment, increases with downstream distance. The reduction in the temporal fluctuations and increase in total entrainment indicates that the streamwise vorticity must therefore play an important role in sustaining the entrainment field of the jet.

Farther downstream the streamwise vorticity becomes even more crucial in the dynamics of the jet. Figures 25 and 26 show velocity vector plots at $x / d=10$ and 15 


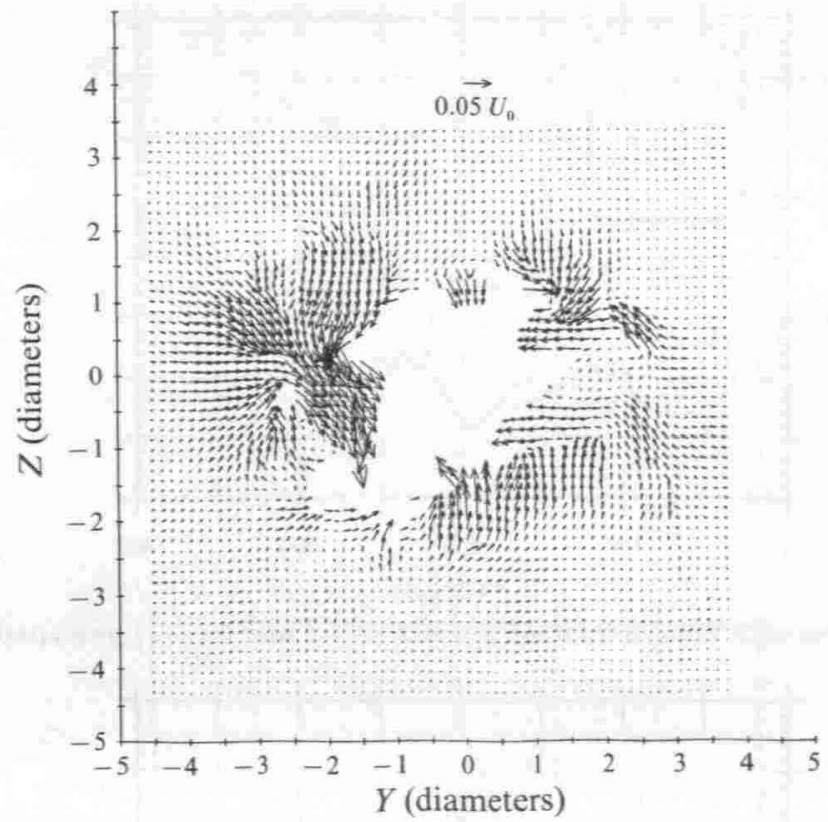

Figure 25. The instantaneous velocity field in a plane normal to the jet axis at $x / d=10$.

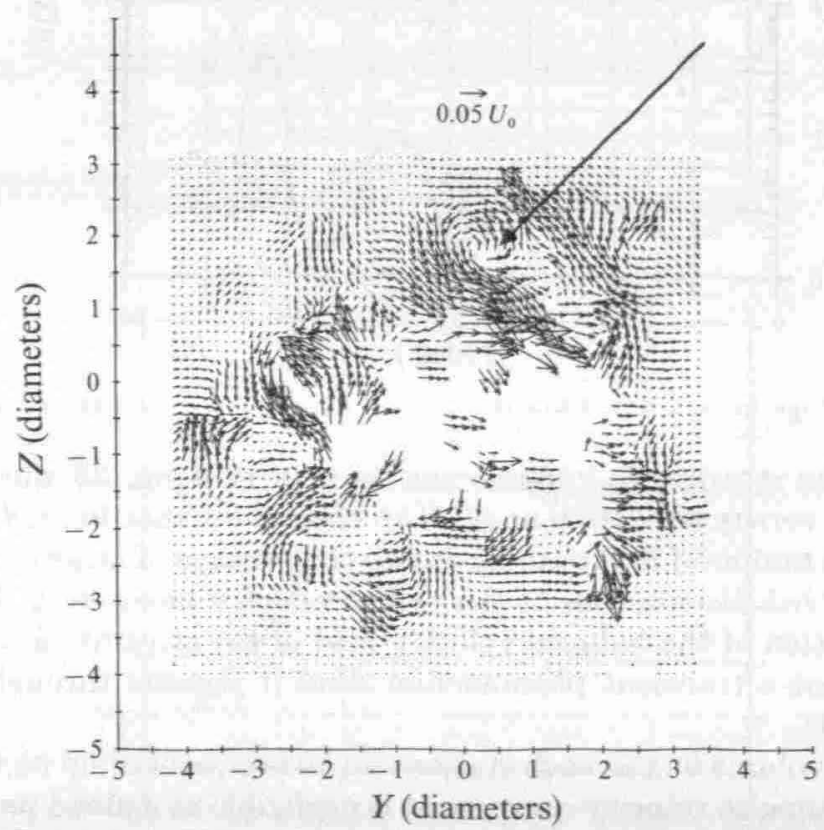

Frgure 26. The instantaneous velocity field in a plane normal to the jet axis at $x / d=15$. The arrow indicates streamwise vorticity with a local strength of $4.25^{-1}$.

respectively. In these images the primary structures no longer entrain evenly around the jet centreline, but the entrainment is controlled predominantly by streamwise vortical structures. One should realize that the primary structures are no longer maintained by the strong velocity difference that exists in the near-field region of the jet $(x / d<5)$. 


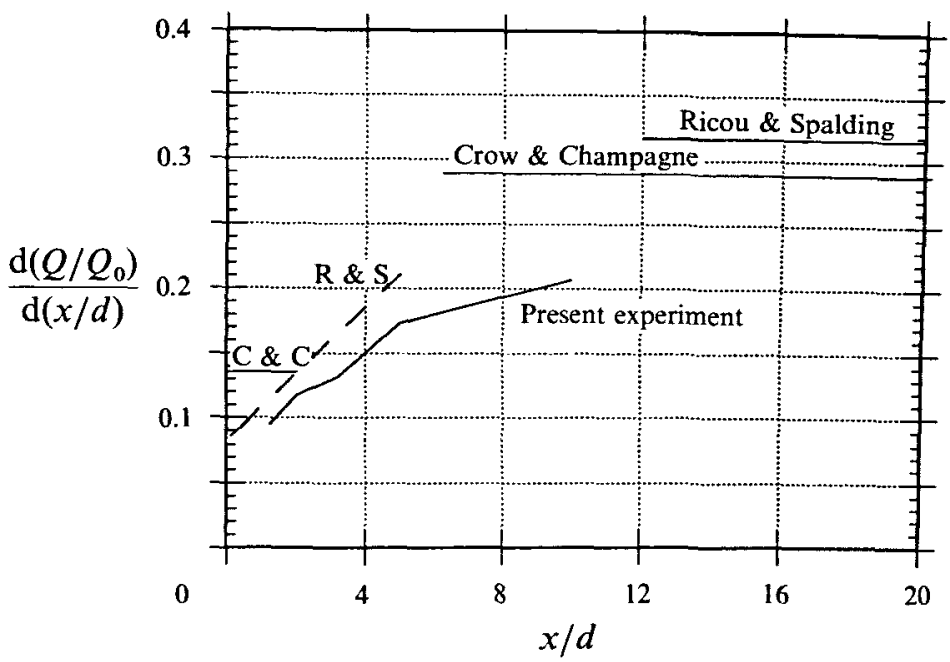

Figure 27. The normalized entrainment versus downstream distance. Suggested curves from Ricou \& Spalding (1961) and Crow \& Champagne (1971) are shown for comparison.

Many investigators have studied the entrainment rate for the near and far field of the round jet. While the entrainment is constant in the far field of self-similar jets, in the near field it must increase from zero at the nozzle to this final rate. As an approximation, Crow \& Champagne (1971) suggested a constant rate for the near field. However, Ricou \& Spalding (1961) suggested a linearly increasing rate for $x / d<5$. Figure 27 shows the entrainment as a function of downstream distance found in this study using the radial volume flux at large $r / d$. The results of the other investigators are also plotted for comparison. The DPIV results indicate an increasing entrainment rate in the near field of the jet with values close to those suggested by Ricou \& Spalding.

\section{Estimate of the strengths of the streamwise vortices}

In the previous section we demonstrated the persistence and the dominant role that the streamwise vortices play in the entrainment field of round jets. It is important to obtain some information about the strengths of these vortices relative to the primary vortical structures. In this section we present a semiquantitative analysis for this purpose.

Any azimuthal perturbation in the form of waviness on the vortex sheet can be amplified by the shear layer. Once there is a radial component of vorticity as in figure 8 , due to small perturbations or waviness, the vorticity is rapidly tilted and stretehed backward. The LIF images of the jet cross-sections around $x / d=2$, where the shear layer first becomes unstable, indicate that the amplitudes of the waves are approximately $2.5 \%$ of the jet diameter. In figure 8 there are nine lobes or waves around the jet with a half-wavelength of approximately $\pi d / 18$. The corresponding radial vorticity due to the tilting of the azimuthal vorticity vector can be estimated by realizing that the local tilting of the vortex lines for the nine lobes can generate a maximum angle $(\alpha)$ of $8^{\circ}$ which corresponds to a $\Omega_{r}$ value of approximately $\sin (\alpha)=0.14 \Omega_{\theta}$. Since in the braid region between the primary rings the thickness of the shear layer and the velocity difference across it is almost the same as at the 
nozzle exit, one can obtain a very conservative estimate of the vorticity in the braid region by dividing the velocity by the momentum thickness, $U_{0} / \delta$. The resulting rate for the tilting of radial vorticity to streamwise vorticity is approximately $(0.14)\left(U_{0} / \delta\right)^{2}$, where $U_{0}$ is the velocity in the potential core and $\delta$ is the momentum thickness at the nozzle. If we non-dimensionalize the expression

$$
[\mathrm{D} \Omega / \mathrm{D} t]_{\zeta} \approx 0.14(U / \delta)^{2}
$$

using $\Omega_{0}=u_{0} / \delta$ and $\delta / U_{0}$ as the characteristic vorticity and time scales we get the transfer rate

$$
\left[\frac{\mathrm{D}\left(\Omega / \Omega_{0}\right)}{\mathrm{D}(t \delta / U)}\right]_{\zeta} \approx 0.14
$$

Even though the amount of the initial perturbation is less than $2.5 \%$ of the jet diameter, the resulting rate at which radial vorticity is transformed to axial vorticity is relatively large.

An investigation of the vorticity at $x / d=15$ showed that circulation associated with streamwise vorticity could approach that of the initial circulation leaving the nozzle. The arrow in figure 26 indicates the location of a streamwise vortical structure which has a local average rorticity of $4.2 \mathrm{~s}^{-1}$. The average vorticity magnitude associated with a primary structure is approximately $5.2 \mathrm{~s}^{-1}$ which can be estimated from the circulation flux from the nozzle, $\frac{1}{2} U_{0}^{2}$, by dividing by the initial frequency and wavelength squared of the primary structures. The actual measurements indicate that the streamwise vorticity in the near field is very active as a result of tilting and stretching of the azimuthal component and supports the entrainment results of the previous section.

\section{Conclusions}

This paper has focused on the formation and growth of streamwise vorticity in the near field of a round jet. Streamwise vortex pairs have a fundamental effect on the entrainment rate and dynamic behaviour of the flow. These findings suggest a central role for the streamwise structures in the dynamics and statistical properties of the shear layer.

The flow visualization experiments show the formation of streamwise vortex pairs, similar to the Bernal-Roshko structures in shear layers, that grow from wave-like instabilities in the early development of the jet after the first rollup of the vortex sheet. The primary structures appear like vortex rings which grow and amalgamate with other axisymmetric structures as they move downstream. During their development, the jet core forms an azimuthal instability in the rortex sheet surrounding the flow. Once the shear layer is no longer round, the small perturbations grow in the highly strained region between two rortex rings.

The results of the DPIV measurements show that the streamwise vorticity does more than just generate three-dimensional motion in the flow. In the near field of the jet, the streamwise vorticity plays a major role in sustaining the inward flow around the jet; this contributes to the continuous growth of the jet. Even small variations in the entrainment field can both instigate and amplify the growth of further secondary instabilities.

After the end of the potential core when the jet is assumed turbulent, at $x / d=10$ and 15 , the DPIV results showed the presence of strong streamwise vorticity. At these locations, the periodic entrainment rates caused by large azimuthal rollers are 
extremely small compared to the entrainment due to streamwise vortex structures. The data indicate that the azimuthal structures lose their energy much faster than the secondary structures and that these dominate the flow field at these locations.

In future work we will study the decay of the streamwise vorticity with downstream distance. We predict a scenario similar to the one described by Cimbala, Nagib \& Roshko (1988) where the primary structures in wake of a circular cylinder decay due to the three-dimensional effects, after which new structures proportional to the local scales of the flow appear farther downstream.

The authors gratefully acknowledge the help of $\mathrm{C}$. Willert for the DPIV experiments. We also acknowledge constructive discussions with G. Broadwell, J. Lasheras, R. Moser, C. Pozrikidis, and A. Roshko. This work has been sponsored by DARPA through the URI program (contract number DARPA/ACMP N00014-86K-0758) and ONR (contract number N00014-89-1529).

\section{REFERENCES}

AgUr, J. C. \& Hesselink, L. 1988 Flow visualization and numerical analysis of a coflowing jet: a three-dimensional approach. J. Fluid Mech. 191, 19-35.

Ashurst, W. T. \& Merburg, E. 1988 Three-dimensional shear layers via vortex dynamics. J. Fluid Mech. 189, 87-116.

BerNaL, L. P. 1981 The coherent structure in turbulent mixing layers. II Secondary streamwise vortex structure. Ph.D. thesis, California Institute of Technology.

Bernal, L. P. \& Roshko, A. 1986 Streamwise vortex structure in plane mixing layers. J. Fluid Mech. 170, 499-525.

Breidenthal, R.E. 1981 Structure in turbulent mixing layers and wakes using a chemical reaction. J. Fluid Mech. 109, 1-24.

BRowaNd, F. K. \& LAUTER, J. 1975 The role of large scale structures in the initial development of a circular jet. In 4th Biennial Symp. on Turbulence in Liquids, University of Missouri-Rolla.

Brows, G. L. \& Roshko, A. 1974 On density effects and large structures in turbulent mixing layers. J. Fluid Mech. 64, 775-816.

Cimbala, J. M., Nagib, H. M. \& Roshko, A. 1988 Large structure in the far wakes of twodimensional bluff bodies. J. Fluid Mech. 190, 265-298.

Corcos, G. M. \& Lrv, S. J. 1984 The mixing layer: deterministic models of a turbulent flow. Part 2. The origin of three-dimensional flow. J. Fluid Mech. 139, 67-95.

Crow, S. C. \& Champagne, F. H. 1971 Orderly structure in jet turbulence. J. Fluid Mech. 48, $547-591$.

Dmotakis, P. E., Mrake-Lye, R. C. \& Papantoniou, D. A. 1983 Structure and dynamics of round turbulent jets. Phys. Fluids 26, 3185-3192.

Gharib, M. \& Willert, C. 1989 Particle tracing revisited. In Advancements in Fluid Mechanic Measurements (ed. MI. Gad-el-Hak), pp. 107-126. Springer.

Jimenez, J. 1983 A spanwise structure in the plane shear layer. J. Fluid Mech. 132, 319-336.

KoNRAD, J. H. 1976 An experimental investigation of mixing in two-dimensional turbulent shear flows with applications to diffusion-limited chemical reactions. Ph.D. thesis, California Institute of Technology.

Lasheras, J. C., Cho, J. S. \& MaXworthy, T. 1986 On the origin and evolution of streamwise vortical structures in a plane, free shear layer. J. Fluid Mech. 172, 231-258.

LIEPMANA, D. 1990 The near-field dynamics and entrainment field of submerged and near-surface jets. Ph.D. thesis, University of California, San Diego.

LIEPyANs, D. 1991 Streamwise vorticity and entrainment in the near field of a round jet. Phys. Fluids A 3, 1179-1187.

Liepnans, D. \& GharIB, M. 1989 The effect of a free surface on the stability of a round jet. Bull. Am. Phys. Soc. 34, 2320. 
Liepuane, H. W. \& Laufer, J. 1947 Investigation of free turbulent mixing. NACA Tech. Note 1258.

LIN, S. J. \& Concos, G. M. 1984 The mixing layer: deterministic models of a turbulent flow. Part 3. The effect of plane strain on the dynamics of streamwise rortices. J. Fluid $\mathbf{1}$ ech. 141, 139-178.

Martin, J. E. \& MeIburg, E. 1991 Numerical investigation of three-dimensionally evolving jets subject to axisymmetric and azimuthal perturbations. J. Fluid Jech. 230, 271-318.

Meiburg, E. \& Lasheras, J. C. 1988 Experimental and numerical investigation of the threedimensional transition in plane wakes. J. Fluid Mech. 190, 1-37.

Meiburg, E., Lasheras, J. C. \& Ashurst, W. T. 1988 Topology of the vorticity field in threedimensional shear layers and wakes. Fluid Dyn. Res. 3, 140-148.

Monkewitz, P. A., Lehman, B., Barsikow, B. \& Bechert, D. W. 1989 The spreading of selfexcited hot jets by side-jets. Phys. Fluids A 1, 446-454.

Ricou, F. P. \& Spalding, D. B. 1961 Measurements of entrainment by axisymmetrical turbulent jets. J. Fluid Ifech. 11, 21-32.

Rogers, M. M. \& Moser, R. D. 1992 The three-dimensional evolution of a plane mixing layer: the Kelvin-Helmholtz rollup. J. Fluid Mech. 243, 183-226.

Widnall, S. E. \& Sullivan, J. P. 1973 On the stability of vortex rings. Proc. R. Soc. Lond. A 332, $335-353$.

Willert, C. E. \& Gharib, M. 1991 Digital particle image velocimetry. Exps. Fluids 10, 181-193.

Winant, C. D. \& Browand, F. K. 1974 Vortex pairing: the mechanism of turbulent mixing-layer growth at moderate Reynolds number. J. Fluid IIech. 63, 237-255.

WygNanski, I. \& Fiedler, H. 1969 Some measurements in the self-preserving jet. J. Fluid Mech. $38,577-612$.

YULE, A.J. 1978 Large-scale structure in the mixing layer of a round jet. J. Fluid Mech. 89, 413-432. 